\title{
El rol del diseño industrial en la movilidad sostenible del D. $M$. Quito
}

\author{
The Role of Industrial Design in the Sustainable Mobility of D. M. Quito
}

Remache A.1; Arroyo F.2
1 Universidad Central del Ecuador, Facultad de Ingeniería, Ciencias Físicas y Matemática. Quito, Ecuador
email: abelbelo@ hotmail.com
2 Universidad de Valladolid, Escuela de Ingenierías Industriales. Valladolid, España
email: flavio.arroyo@gmail.com
Información del artículo
Recibido: enero 2020
Aceptado: marzo 2020

\section{RESUMEN}

El presente artículo analiza la movilidad sostenible, sus características, su relación con el diseño industrial, ventajas y puntos clave de enfoque para la concepción de esta en la Ciudad de Quito, especialmente el proyecto de movilidad Metro. Con las características de las Smart City, se busca el desarrollo de la sociedad estableciendo un equilibrio ambiental, social y económico y utiliza la tecnología como aliado para poder sustentar su desarrollo analizando los diferentes proyectos de transporte implementados en las ciudades más desarrolladas, presentándose como el mayor problema que se atraviesa, y para ello el diseño industrial surge como respuesta para lograr construir una ciudad inteligente enfocada en mejorar la toma de decisiones, la eficiencia de las operaciones y procesos de innovación, la prestación de los productos y servicios urbanos y su competitividad gracias a que es capaz de comprender los procesos tecnológicos, sociales y del entorno desde una perspectiva sistémica, en la que las relaciones constituyen la base de su acción, brindando soluciones claras y rentables para transformar las ciudades actuales en Smart Cities. El metro de Quito actualmente es el proyecto más importante dentro del área de movilidad de la ciudad, el cual impone un nuevo hito hacia la prosperidad en el desarrollo de las ciudades inteligentes de Latinoamérica, otorgando una facilidad para movilizarse en los medios de transporte, reducción del tránsito vehicular, mayor acogida de extranjeros y propios y logrando el contento y comodidad de sus residentes y ciudadanos en esta nueva era.

Palabras Clave: Movilidad sostenible, transporte, desarrollo sostenible, diseño industrial, Smart cities

\begin{abstract}
This article analyzes the mobily sustainability, their characteristics, their relationship with industrial design, advantages and key points of focus for the design of this in the City of Quito, especially the Metro mobility project. With the characteristics of the Smart City, the development of society is sought by establishing an environmental, social and economic balance and uses technology as an ally to sustain its development by analyzing the different transport projects implemented in the most developed cities, presenting itself as the biggest problem that is going through, and for this industrial design arises as a response to build an intelligent city focused on improving decision making, the efficiency of operations and innovation processes, the provision of urban products and services and their competitiveness thanks to its ability to understand technological, social and environmental processes from a systemic perspective, in which relationships are the basis of its action, providing clear and profitable solutions to transform current cities into Smart Cities. The Quito metro is currently the most important project within the area of mobility of the city, which imposes a new milestone towards prosperity in the development of smart cities in Latin America, providing a facility to mobilize in means of transportation, reduction of vehicular traffic, greater reception of foreigners and their own and achieving the contentment and comfort of its residents and citizens in this new era.
\end{abstract}

Keywords: Sustainable mobility, transport, sustainable development, industrial design, Smart cities 


\section{Introducción}

El objetivo principal es analizar el desarrollo de las ciudades inteligentes en la actualidad, tomando en cuenta el diseño industrial como punto de enfoque y verificar que en este sentido pueda adaptarse en el Distrito Metropolitano de Quito, especialmente en el proyecto de movilidad "Metro de Quito".

En todo el mundo el crecimiento de la población urbana es exponencial y a medida que aumenta aparecen nuevos problemas y necesidades que satisfacer dentro del desarrollo de las ciudades, como las soluciones europeas y asiáticas más destacables. Con las características de las Smart City, se busca el desarrollo de la sociedad estableciendo un equilibrio ambiental, social y económico y utiliza la tecnología como aliado para poder sustentar su desarrollo analizando los diferentes proyectos de transporte implementados en las ciudades más desarrolladas, presentándose como el mayor problema que se atraviesa, y para ello el diseño industrial surge como respuesta para lograr construir una ciudad inteligente enfocada en mejorar la toma de decisiones, la eficiencia de las operaciones y procesos de innovación, la prestación de los productos y servicios urbanos y su competitividad gracias a que es capaz de comprender los procesos tecnológicos, sociales y del entorno desde una perspectiva sistémica, en la que las relaciones constituyen la base de su acción, brindando soluciones claras y rentables para transformar las ciudades actuales en Smart Cities.

El diseño de políticas y sistemas de movilidad urbana se ha convertido en un problema clave de investigación e intervención para los gobiernos y otras partes interesadas en promover los objetivos de sostenibilidad. La movilidad sostenible es un desafío relevante abordado hoy por los líderes políticos, gerentes públicos e investigadores de todo el mundo. La movilidad sostenible es un término que resume lo que está en juego en los intentos contemporáneos de corregir el equilibrio de costos y beneficios en el sector del transporte [1] [2] [3] [4] [5] [6].

Concretamente la metodología de Diseño para la Sustentabilidad (D4S) juega un papel muy importante cuando se asocia a modelos de producción sostenibles, considerando el desarrollo del producto como un sistema integrado donde cada decisión influye en todo el proceso y produce dife- rentes impactos en el medio ambiente. El D4S consiste básicamente en innovaciones tecnológicas y procedimientos metodológicos que tienen como objetivo ayudar a los diseñadores y tomadores de decisiones a producir bienes y servicios económicamente viables y ecológicos [7] [8].

Por otra parte, la movilidad sostenible es el modelo de movilidad que permite el movimiento con un impacto ambiental y territorial mínimo [9] [10] [11]. William R. Black, da una definición correcta sobre transporte sostenible genera controversias y desacuerdos, ya que, según él, sería la capacidad de satisfacer las necesidades de transporte actuales sin comprometer la capacidad de las generaciones futuras para satisfacer estas necesidades [12]. Claus Doll y Martin Wietschel añade que los sistemas de transporte realizan las funciones vitales para la sociedad, pero en su estado actual no puede ser considerar sistemas sostenibles. Preocupaciones particulares en este sentido incluyen el cambio climático, las emisiones locales de aire, el ruido, la congestión y los accidentes [13]. Los impactos negativos del sector del transporte en el medio ambiente también son cada vez más evidentes [14] [15].

El transporte es un factor importante en el contexto del desarrollo sostenible debido a la presión que ejerce sobre el medio ambiente, sus impactos económicos y sociales y sus vínculos con otros sectores.

La gobernanza sostenible de los sistemas de transporte sigue siendo un desafío importante para los responsables políticos de todo el mundo, especialmente en las ciudades. Las áreas urbanas se están desarrollando rápidamente desde un punto de vista tecnológico, y las tecnologías innovadoras crean nuevas posibilidades para la gestión inteligente de la movilidad. Se espera que la implementación del concepto de ciudad inteligente exhiba un alto potencial en términos de movilidad sostenible y mitigación de emisiones [16][17].

La movilidad inteligente es un concepto amplio que facilita el logro de un desarrollo sostenible mediante la optimización de los servicios de transporte, teniendo en cuenta los desafíos tecnológicos, sociales, económicos y ambientales. La protección del medio ambiente es un aspecto importante de diversas políticas mundiales y europeas. La movilidad con bajas emisiones es una parte esencial del cambio más amplio hacia la economía circular baja en carbono necesaria para satisfacer las necesida- 
des de movilidad de las personas y el transporte de mercancías. Para garantizar la mejora deseada en la movilidad, se emprendieron muchas iniciativas en los últimos años[18].

El Distrito Metropolitano de Quito (DMQ) con una población aproximada de 2'239.191 habitantes, según el informe de movilidad genera un 4' 600.000 desplazamientos, que pueden ser utilizados de en diferente modalidad. Se demuestra que el modo preferencial de desplazamiento de los habitantes en el DMQ es el transporte público, que en términos generales representa el 61,3\% y con relación a los modos motorizados el 73\%. [19].

El aire ha sido uno de los recursos que más ha sufrido contaminación debido al incremento poblacional y económico de las ciudades. Es así que la principal fuente de contamínate del aire de Quito son las fuentes móviles, es decir la contaminación que causan los vehículos motorizados en las emisiones que se dan a partir de la combustión de la gasolina de cualquier tipo y diésel. El incremento del parque automotor en la ciudad, incremento de circulación y tiempos de viaje son los factores fundamentales en la generación de contaminantes a la atmosfera de Quito. Según el Ministerio de Ambiente el $76 \%$ de la contaminación proviene de los automóviles y esto ha tenido consecuencias respiratorias a más de 1012 millones de personas [20].

Los mapas de emisión del tráfico, se estructuran típicamente en base de mapas de intensidad de tráfico como muestra lafigura.

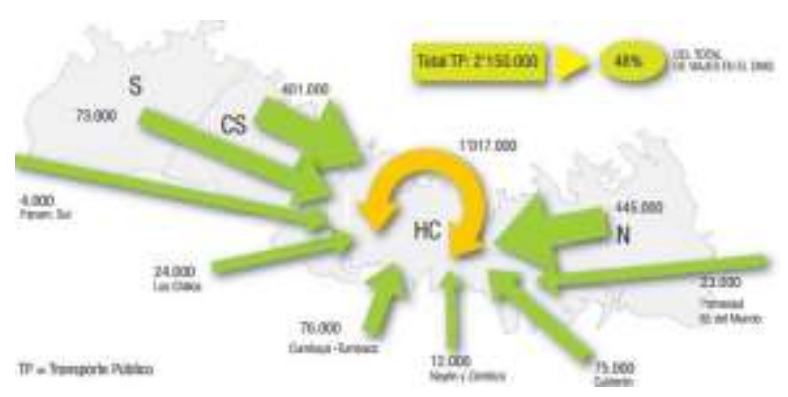

Figura 1. Número de desplazamientos motorizados al día en Quito [21]

\section{Método}

El presente trabajo emplea una metodología basada en la Investigación Científica y el Método Deductivo Indirecto, el cual permitió la deducción de conclusiones acerca de la temática tratada en el de- sarrollo del documento, esta metodología fue empleada para el análisis de la movilidad sostenible y cómo el diseño industrial actúa como herramienta clave para el desarrollo de productos y servicios con el fin de construir una ciudad inteligente enfocada en la movilidad de sus ciudadanos.

\subsection{Movilidad Smart}

Según el Libro Blanco Smart Cities [22], la estructura de una Smart City está conformada por los siguientes elementos: Espacio urbano, Sistema de infraestructuras, Complejo de redes y plataformas inteligentes y Ciudadanía que ejerza de eje vertebrador.

Así mismo, el libro blanco recomienda aplicar principios que estén articulados entre sí, tales como: La infraestructura tecnológica: redes de información como mecanismo de comunicación, plataformas inteligentes, infraestructuras ecoeficientes, etc.; la estrategia energética: uso de energías renovables, sistemas de almacenamiento y aprovechamiento de energía, etc.; la gestión y protección de los recursos: ordenación del territorio y de los recursos basada en criterios de sostenibilidad, cooperación entre administraciones, etc.; la provisión de servicios: desarrollo de nuevos modelos colaborativos que permitan integrar lo público y lo privado, modelos de servicios mancomunados, etc.; el Gobierno: accesibilidad de los datos, transparencia en la gestión, aplicación de políticas sostenibles, entre otras [22].

\subsection{El diseño Industrial en la movilidad}

Una forma de materializar el concepto de sostenibilidad es a través del estudio de los indicadores ambientales en el contexto de la movilidad urbana juegan un papel clave en la evaluación de la influencia de los diferentes modos de transporte, ya sean individuales o colectivos, motorizados o no motorizados, y en la reducción de la contaminación del aire, entre otros asuntos relacionados a la protección del medio ambiente. Los indicadores sociales también son útiles para evaluar la mejora de las condiciones de desplazamiento de la población en las zonas urbanas, incluida la proximidad a los servicios de transporte, la comodidad y la puntualidad [15].

El objetivo primordial del diseñador dentro de las smart cities es humanizar la tecnología utilizada 
para conectar a los humanos entre sí y que esta sea lo más cercana posible a su entorno para que no le parezca extraña y la adopte con naturalidad. Además, el diseñador se convierte en un actor importante en el proceso de ayudar a la comunidad a generar respuestas que le ayuden a solventar sus problemas con metodologías participativas aplicadas en la innovación social. [23].

\subsection{Proyectos de movilidad}

A nivel Nacional podemos analizar los pilares del Plan Nacional de seguridad Vial establecido en 2013 y que sigue en vigencia actualmente, cuyos objetivos se verían cubiertos en los resultados de una ciudad inteligente. Este plan busca establecer la obligatoriedad de la aplicación de parámetros de seguridad definidos por MTOP, en el desarrollo de proyectos integrales de infraestructura vial, impulsar la aplicación de mejores tecnologías de seguridad pasiva y activa de los vehículos, promover una mejora continua en el comportamiento de los usuarios de las vías, garantizar una atención integral, oportuna y óptima a las víctimas de los siniestros. [24].

El DMQ ha implementado en su sistema de transporte varios medios de movilización, en donde, el transporte de buses colectivos es el más utilizado por al menos el 70\% de la población e incluye: Sistema Trolebús, la Ecovía, el corredor Central Norte y el corredor Sur Oriental. Según Vizcarra (2010) el sistema de transporte está integrado por buses y colectivos urbanos que tienen 134 líneas convencionales de transporte público operadas por 2136 buses urbanos y buses interparroquiales compuestos por 46 líneas operadas por 500 buses de servicio micro regional[25].

Otra medida de movilidad es el denominado Pico y placa, siendo esta una medida de restricción vehicular implementada inicialmente en la ciudad de Bogotá. Es una medida de gestión de la demanda de transporte para racionar el uso de una escasa oferta de transporte ante una demanda excesiva. Esta norma de tránsito impone una restricción de circulación obligatoria en el área urbana a vehículos privados tipo automóvil y de servicio público en horarios "pico" (horarios con mayor afluencia de tráfico), dependiendo del último número de placa el automóvil, pretendiendo reducir con ella el colapso circulatorio que se formaba en estas horas. Dentro de su aplicación, cada año se rota el día de restricción de acuerdo al número de placa del vehículo. [26]. El Pico y Placa comprende las avenidas Morán Valverde, al sur; Diego de Vásquez, al norte; Mariscal Sucre, al occidente; y Simón Bolívar, al oriente. La restricción será de 07:00 a 09:30 y de 16:00 a 19:30. La medida forma parte de un conjunto de acciones que aplica el Cabildo para mejorar la movilidad [27].

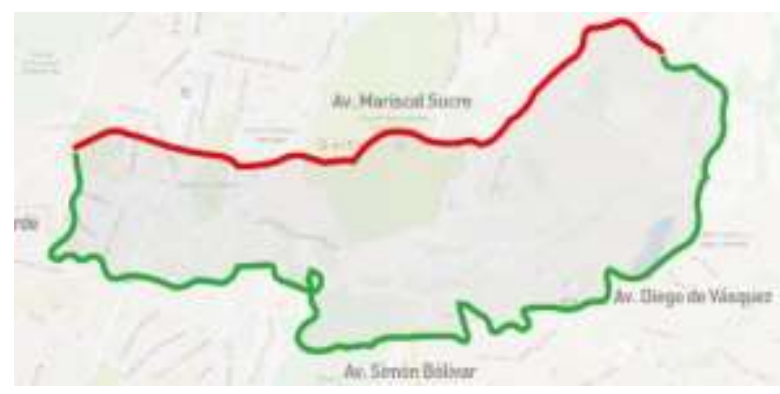

Figura 2. Perímetro de aplicación ordenanza Pico y Placa [27]

El 9 de octubre del 2019 entró en vigencia la restricción vehicular "hoy no circula" reemplazando el "pico y Placa", si bien esta ordenanza no tiene como objetivo principal resolver el tema del tránsito en el D. M. Quito, se podría analizar los resultados que se tenga a mediado plazo.

El sistema de transporte público "Ecovía" fue el segundo sistema de transporte implementado en Quito. Comenzó su funcionamiento en 2001 en el tramo comprendido entre el Playón de la Marín y la estación Río Coca. Este corredor se complementó en 2011 con la apertura de la extensión Sur Oriental, que hoy tiene conexión con la terminal Quitumbe y con la nueva Terminal Sur Ecovía [28]. El sistema de transporte corredor Sur Occidental, se implementó en 2012, diseñado para brindar servicio y complementar el transporte en los barrios del sur del Distrito. Este sistema cuenta con un corredor exclusivo que une Quitumbe con la terminal de transferencia ubicada en el Seminario Mayor. Desde allí, los usuarios pueden trasladarse al corredor central norte, MetrobúsQ, y llegar hasta el sector de la Ofelia en el noroccidente de la ciudad. Adicionalmente, el sistema municipal de transporte administrado por la EPMTPQ cuenta con más de 40 líneas alimentadoras y de integración, las cuales amplían su cobertura y ofrecen a los usuarios alternativas económicas para movilizarse y cumplir con sus actividades cotidianas. Cada día, 
la Empresa transporta alrededor de un millón de pasajeros en todos sus circuitos y rutas. [28]

Otro esfuerzo que se ha gestionado se ve en la integración de un bus eléctrico (Cero gases contaminantes) que empezó a circular en el DMQ desde el 11 de diciembre de 2017, con una longitud de 18 metros, capacidad para 160 pasajeros, y limita su velocidad a 60 kilómetros por hora. [29]. El bus tiene una potencia de $360 \mathrm{KW} / \mathrm{h}$, equivalente a 482 HP (caballos de fuerza); sus baterías se recargan en 3 horas, y su vida útil es de 15 años [30]. Se ha propuesto que el sistema de transporte público al 2020 dentro del DMQ tenga un transporte público con cero emisiones de carbono [31].

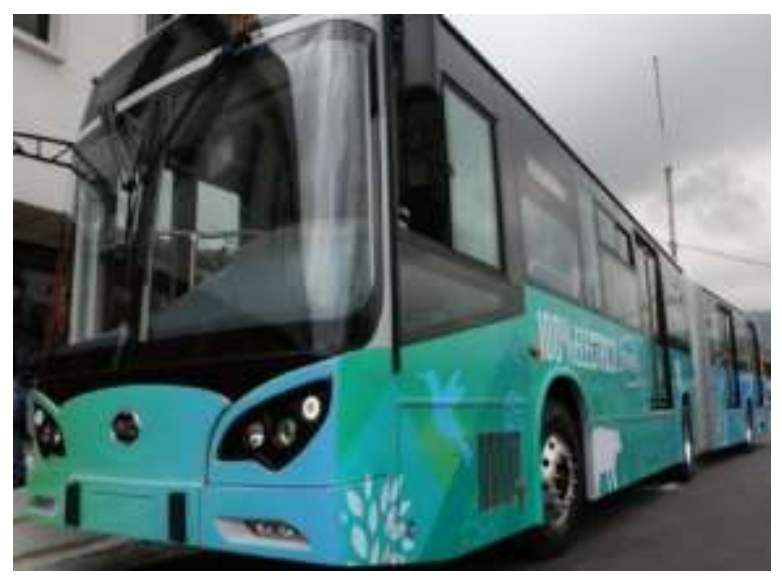

Figura 3. Primer bus eléctrico del Ecuador [30]

BICIQUITO considerada como la Bicicleta Pública de Quito está disposición de los usuarios, y ha sido una implementación basada en varias experiencias generadas en otros países. El sistema consiste en el préstamo gratuito de las bicicletas para transportarse dentro del perímetro establecido [32].

Existe un plan futuro que consiste en la construcción de un sistema de monorriel desde el valle de los Chillos hasta el DMQ, en donde se pretende beneficiar a más de 300000 usuarios [33].

\section{Discusión y Resultados}

En el Distrito Metropolitano de Quito, el transporte público (buses del sistema metro-Q, buses urbanos e interparroquiales) representa el $62.8 \%$ del total de medios de transporte utilizados por los ciudadanos para movilizarse [34]. El parque automotor en el DMQ en el factor de mayor inciden- cia en el incremento de las congestiones de tráfico, cada vez más severas durante los períodos pico del día de manera especial en el hipercentro de la ciudad, evidenciando estos inconvenientes en la red vial principal de los Valles de los Chillos, Tumbaco y Cumbayá [35].

La demanda de transporte masivo e individual va relacionada con el crecimiento demográfico acelerado en los centros urbanos. El tráfico y la movilidad, en términos de transporte, son la causa principal de los impactos negativos al ambiente urbano como la contaminación del aire, el ruido, el consumo excesivo de recursos y la ocupación extensiva del espacio. El parque vehicular del DMQ esta predominado por lo automotores livianos que evidencia una tendencia en alza en propiedad de vehículos por habitante[35].

Cada ciudad muestra como está funcionando cada sociedad internamente, y las ciudades comúnmente albergan o concentran grandes grupos humanos tal como muestran los últimos informes de la ONU, en donde se prevé que en el año 2050 las ciudades concentraran al $70 \%$ de la población mundial [22]. En la siguiente figura se puede evidenciar que el crecimiento de la población en las ciudades y en el mundo es exponencial:

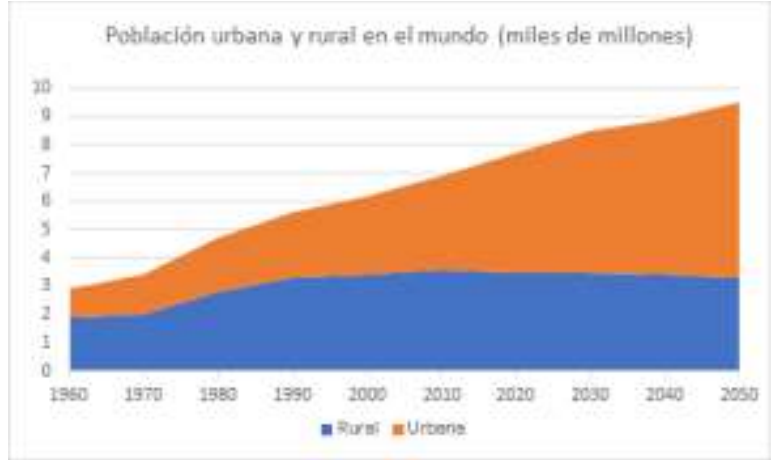

Figura 4. El aumento a nivel mundial de los habitantes de las ciudades [36].

En el planeta, las ciudades representan apenas el $2 \%$ del espacio, pero el consumo de energía corresponde al $60 \%$ al $80 \%$ del mundo y generan aproximadamente el $75 \%$ de las emisiones de carbono. En las ciudades de América Latina y El Caribe, se estima que concentran el 36\% de la población total [37]. Así como puede ser impresionante la concentración demográfica también puede representar un escenario necesario para implementar la idea 
de las Smart Cities, o crear una nueva ciudad. Esto con el afán y visión de la apertura de nuevos negocios. En la siguiente gráfica se puede observar el crecimiento de las Smart Cities por sector.

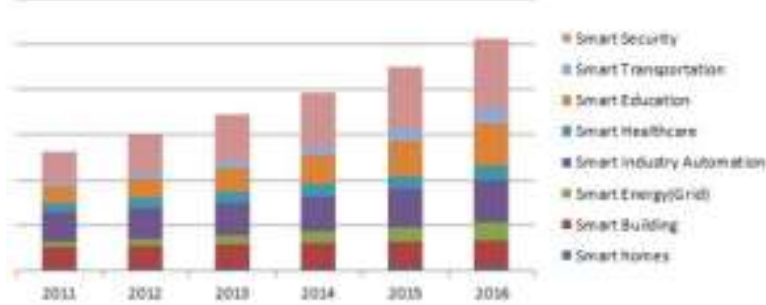

Figura 5. Crecimiento del mercado de Smart Cities por sector, según el informe de Markets and Markets [22]

Se afirma que en el año 2017 se mal gastaban casi € 300 millones en energía, en comida desperdiciada alrededor de $€ 150$ millones, pérdidas por congestiones $€ 100$, y la muerte de 7 millones de personas por causa de la polución. Se cree que una ciudad inteligente debe basar su tecnología y sus aparatos de uso cotidiano a combatir estas principales pérdidas de manera ordenada y sabia [38].

Una Smart City se esfuerza por promover la seguridad a todo nivel, la sostenibilidad, la mejora y optimización de las infraestructuras y los sistemas de transporte en todas las modalidades. Uno de los principales retos en la actualidad de una Ciudad Inteligente se manifiesta en el control del tráfico con el fin de mejorar varias consecuencias que conlleva el no tener una buena gestión en este ámbito tales como son la productividad, uso de combustible, niveles de emisiones de carbono, enfermedades, entre otras. Las Smart Cities impulsan el uso y cambio de matrices energéticas renovables y modos de transportes sustentables (energía limpia) [39]. La mejora del transporte público es un factor extremadamente fundamental para la movilidad sostenible y la optimización del uso del tiempo [40].

El transporte público representa un eje fundamental para vertebrar la estrategia de movilidad de una Smart City, ya que, en sus distintas vertientes (autobús, suburbano, tren), aventaja claramente en términos de sostenibilidad y eficiencia energética al transporte privado. Tal y como se recoge en el siguiente gráfico, desde el punto de vista del consumo energético por viajero y por Km, el transporte públicoes aproximadamente 6 veces más eficiente que el vehículoprivado:

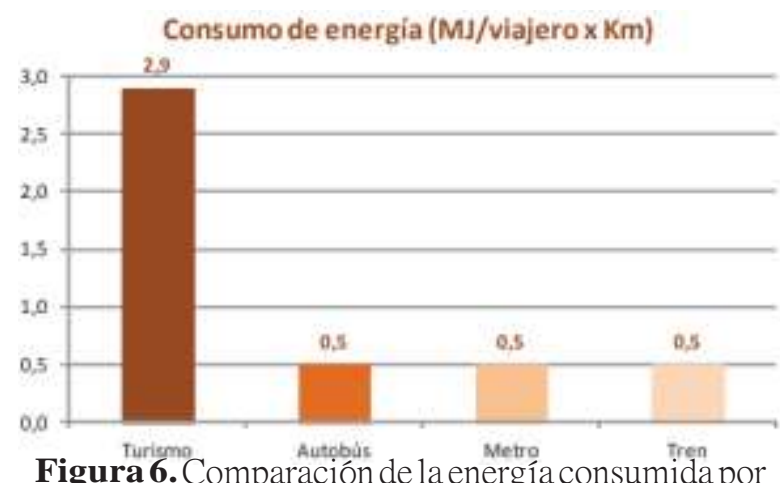

Figura 6. Comparación de la energía consumida por viajero y kilómetro de recorrido, en función del medio de transporte [22]

ElMunicipio de Quito apoyará su planificación de ciudad a través de "Big Data una extensa y variada base de datos que se obtienen a través de la información que generan los usuarios de telefonía celular y a través de los cuales se pueden crear soluciones en torno a la movilidad que se desarrollará en conjunto entre el Municipio de Quito, el Banco Interamericano de Desarrollo (BID) y Telefónica Movistar. Esta permitirá establecer lineamientos para articular servicios para la ciudadanía, como lo hacen las Smart Cities. Que permitirá el desarrollo de política pública y obras dirigidas hacia la comunidad [41].

Además, permitirá analizar datos en tiempo real y de forma dinámica y con ello modelar proyectos que mejoren la movilidad, conociendo cómo se mueven las personas, los puntos críticos de embotellamiento y otros datos que confluyen en la movilidad [42].

Actualmente Quito cuenta con un plan de Movilidad Sostenible, delineado por la construcción de obras significativas como las Ciclovías, Quito Cables, BiciQuito, los Buses eléctricos, la aplicación MovilizateUIO y el Metro de Quito, que demuestran el verdadero interés de transformar a Quito en una ciudad ejemplo en temas de movilidad sostenible [43].

Metro de Quito abre la posibilidad de repensar la ciudad, construir un esquema integral de desarrollo urbano en torno a esta gran obra, contar con esta herramienta será fundamental para hacerla de manera más eficaz [44]. El metro está planificado como el eje central del Sistema Integrado de Transporte de Pasajeros (SITP) del Distrito Metropolitano de Quito (DMQ). Está previsto que el 
SITP lo componen, además del tren subterráneo, el sistema de alimentadores, el transporte convencional, el servicio interparroquial, el Trolebús y los corredores municipales. De acuerdo con el plan, el sistema integrado de transportación permitiría que el $93 \%$ de los usuarios de servicio de transporte público hallen una parada a menos de 4 cuadras de su hogar o lugar de trabajo [44].

En el mundo existen varios ejemplos de cómo el diseño industrial ha desarrollado productos de movilidad con un enfoque al servicio público, mejorando la calidad de vida de los ciudadanos y promoviendo un equilibrio entre el medioambiente y el crecimiento de unaciudad.

Un vehículo representa para la ingeniería de transporte la unidad básica sobre la cual se diseñan, calculan y planean los sistemas de transporte público, con el objetivo de satisfacer las necesidades de una población en condiciones aceptables de confort, confiabilidad y eficiencia. En el caso del diseño automotriz, este vehículo es el objeto que desarrolla teniendo en cuenta necesidades humanas, factores funcionales, prestaciones al usuario, características que permitan su producción en entornos industriales y conceptos estéticos y conceptuales que le dan la forma y la materialidad [45].

De igual forma es un objeto de diseño cuyo objetivo es transportar a distintas personas, sin que estas tengan la intención y la necesidad de comprarlo, a diferencia de cualquier otro tipo de vehículo, que se diseña incluso, pensando en cosas distintas a "transportar", pues el vehículo de transporte público se diseña pensando en transportar, lo que convierte a su comprador en una persona distinta a su usuario [45].

Los usuarios de los sistemas de transporte público urbano, son pasajeros transportados y son personas que necesitan desplazarse en un vehículo público y tienen unos requerimientos operacionales del sistema por los cuales están dispuestos a pagar, convirtiéndolos en sujetos que pueden ser cuantificados y que demandan ciertas características de estos sistemas.

Es necesario diseñar ciudades donde los peatones puedan andar más e ir más en transporte alterno y tener acceso a transporte público que esté a solo 10 minutos caminando desde un punto de partida. Es necesario un plan integrado para poder saber cuándo va a venir un bus y transferencias integradas para no pagar dos veces, para crear un mejor uso de los vehículos [46].

\section{Conclusiones}

Las ciudades constituyen una importante amenaza para el ecosistema del planeta; las cifras muestran un crecimiento exponencial de la población y en el área urbana, pero al mismo tiempo, al tratarse de los mayores polos de concentración de talento, conocimiento y capacidad de innovación, por lo que conviene empezar a trabajar en las oportunidades para contribuir a nuestra calidad de vida y bienestar social a partir de la conversión de ciudades en Smart Cities.

El fin de la tecnología dentro de las Smart Cities es que la gente hable el mismo lenguaje, que las ciudades se hagan entender de la misma manera para los lugareños y visitantes, es generar comunicación efectiva, por lo que el diseño industrial se convierte en un articulador de conocimientos de diferentes disciplinas que traduce a un lenguaje entendible por todos, demuestra el poder de la acción colectiva para resolver problemas desde la inclusión, la sostenibilidad y la resiliencia y, sobre todo, unifica bajo un objetivo común.

El diseño es difícil de medir. Pero puede destacar por la superior calidad de un diseño. Grandes urbes, tienen en el diseño urbano, su valor diferenciador, potenciarlo, agrandarlo y aumentar su valor, genera un beneficio no percibido en los ciudadanos. Se debe promover la participación y la innovación en el diseño urbano. Para inventar y alcanzar un de futuro con capacidad de crear ventajas competitivas. No cabe duda de que las Smart Cities son un sector fértil para la innovación, un espacio abierto donde las propuestas y categorías de servicios y surgen productos. Este campo abre oportunidades y nuevos desafíos del mercado para el diseño industrial y para empresas.

El diseño industrial claramente tiene una fuerte presencia en el metro de Quito, ya que cada vagón está netamente diseñado para la comodidad y flujo de los pasajeros. Uno de los mayores problemas de la ciudad de Quito es la mobilidad, y con este proyecto podemos evidenciar como el diseño industrial presenta una solución atractiva y moderna que cumple con el objetivo de construir una 
ciudad inteligente enfocada en la movilidad de sus ciudadanos.

El lograr construir una ciudad Inteligente es un duro trabajo, que con las herramientas y metodologías necesarias nos facilitara llegar a nuestro objetivo. Una ciudad sostenible es el objetivo de muchos países ya que con todos los cambios climáticos que se están dando en la actualidad el desarrollo tecnológico con un enfoque sostenible es la única forma de actuar para prevenir futuros problemas ambientales, sociales y económicos.

Aplicar el modelo de smart city es una solución que debe considerar la ciudad de Quito, sobre todo para mejorar en cuanto a destino turístico. Aunque es imposible convertirse en ciudad inteligente de forma inesperada y es un proceso extenso y con resultados a largo plazo, ser una smart city significa, mejorar la gestión de la movilidad ayudando a ofrecer una experiencia óptima a turistas y ciudadanos. - La ciudad vista la necesidad de avanzar, innovar y modernizarse, ya ha llevado a cabo algunos proyectos propios de una smart city que están relacionadas con la mejora sostenible, la gestión y participación ciudadana, la transparencia del gobierno y el uso de las nuevas tecnologías de la información y las comunicaciones.

\section{Referencias}

[1] L. Giorgi, «Sustainable mobility. Challenges, opportunities and conflicts - a social science perspective,» International Social Scienc e Journal, pp. 179-183, 2004.

[2] United Nations Centre for Regional Development, Mobility and NMT in Sustainable Urban Development - Role of City, Ulaanbaatar: UNCRD, 2018.

[3] OECD, Managing the Fundamental Drivers of Transport Demand, París: ECMT Publications, 2003.

[4] OECD, «Towards Green Growth: Monitoring Progress,» OECD, PAris, 2011.

[5] G. Santos, H. Behrendt y A. Teytelboym, «Part II: Policy instruments for sustainable road transport,» Research in Transportation Economics, pp. 46-91, 2010.
[6] B. Elzen, F. W. Geels y K. Green, System Innovation and the Transition to Sustainability: Theory, Evidence and Policy, Cheltenham: Edward Elgar Publishing, 2004.

[7] UNEP, Design for Sustainability, País: UNEP, 2006.

[8] H. B. de Barros Pereira y P. F. de Almeida Souza, «Design for Sustainability:,» 5th European Academy of Design Conference, pp. 1-17, 2003.

[9] T. Goldman y R. Gorham, «Sustainable urban transport: Four innovative directions,» Technology in Society, pp. 261-273, 2006.

[10] P. Nijkamp, U. B. Erik Verhoef y C. Rodenburg, «Sustainable mobility,» Transportation engineering and planning, pp. 1-10, 2001.

[11] P. Nieuwenhuis, P. Vergragt y P. Wells, The Business of Sustainable Mobility: From Vision to Reality, New York: Routledge, 2017.

[12] W. R. Black, «Socio-economic barriers to sustainable transport,» Journal of Transport Geography, pp. 141-147, 2000.

[13] C. Doll y M. Wietschel, «Externalities of the transport sector and the role of hydrogen in a sustainable transport vision,» Energy Policy, pp. 4069-4078, 2008.

[14] D. Banister, «Cities, mobility and climate change,» Journal of Transport Geography, pp. 1538-1546, 2011.

[15] J. M. López Pol y M. d. V. Segarra-Oña, «Smart Cities: nuevos focos de innovación para un desarrollo sostenible,» Universidad Politécnica de Valencia, pp. 1-29, 2013.

[16] P. Neirotti, A. De Marco, A. C. Cagliano, G. Mangano y F. Scorrano, «Current trends in Smart City initiatives: Some stylised facts,» Cities, pp. 25-36, 2014.

[17] T. Nam y T. A. Pardo, «Conceptualizing Smart City with Dimensions of Technology, People, and Institutions,» The Proceedings of the 12th Annual International Conference on Digital Government Research, pp. 282-291, 2011. 
[18] J. Zawieska y P. Jana, «Smart city as a tool for sustainable mobility and transport decarbonisation,» Transport Policy, pp. 3950, 2018.

[19] S.d.Movilidad, «MUNICIPIODEL DISTRITO METROPOLITANO DE QUITO,» 2016. [En línea]. Available: http:// gobiernoabierto.quito.gob.ec/wp-content/ uploads/documentos/pdf/diagnosticomovilidad.pdf.

[20] F. J. Carphio Martínez, «UDLA,» 2015. [En línea]. Available: http://dspace.udla. edu.ec/handle/33000/3656.

[21] M. D. D. M. D. QUITO, «PLAN MAESTRO DE MOVILIDAD,» 2010. [En línea]. Available: http://www.flacsoandes. edu.ec/libros/118765-opac.

[22] Enerlis; Ernst; Young, «LIBRO BLANCO SMART CITIES,» Septiembre 2012. [En línea]. Available: http://www.innopro.es/ pdfs/libro_blanco_smart_cities.pdf.

[23] D. Cárdenas y F. Camargo, «Las smart cities, la comunicación y el diseño: un reto de la sociedad del conocimiento.,»Virtu@ lMente, pp. 42-55, 2016.

[24] ANT, «Plan Nacional de Seguridad Vial,» Agencia Nacional de Tránsito,2013.

[25] J. S. Vizcarra, 2010. [En línea]. Available: https://www.puce.edu.ec/documentos/ opsa/Movilidad.pdf.

[26] L. Hora, «La Hora,» 0105 2010. [En línea]. Available: https://lahora.com.ec/noticia/1028329/pico-y-placa-guc3ada--paralos-carros-que--vienen-a-la-ciudad.

[27] A. M. d. Transito, 2018. [En línea]. Available: http://www.amt.gob.ec/index.php/ pico-placa-homepage.html.

[28] EPMTP, 1503 2017. [En línea]. Available: http://www.trolebus.gob.ec/index.php/sobre-nosotros/historia-institucional.

[29] M. B. Merizalde, 3011 2017. [En línea]. Available: http://www.elcomercio.com/ actualidad/quito-primer-bus-electrico-transporte.html.
[30] Quitoinforma, 0112 2017. [En línea]. Available: http://www.quitoinforma.gob. ec/2017/12/01/el-primer-bus-articulado-electrico-ya-circula-en-quito/.

[31] A. Almeida, 0112 2017. [En línea]. Available: http://www.extra.ec/actualidad/ buselectrico-quito-transporte-publi- coBG1877892.

[32] BiciQuito, 2017. [En línea]. Available: http://www.biciquito.gob.ec/index.php/ info/que-es.html.

[33] G. M. d. Rumiñahui, 1901 2017. [En línea]. Available: http://www.ruminahui.gob.ec/index.php?option $=$ com content\&view $=$ article $\& i d=374: \&$ catid $=8 \&$ Itemid $=113 \&$ lang $=\mathrm{kw}$.

[34] Distrito Metropolitano de Quito, «Plan maestro de movilidad para el Distrito Metropolitano de Quito: 2009-2025,» Distrito Metropolitano de Quito, Quito, 2009.

[35] RCP Consultores Asociados, «Consultoría a fin de desarrollar los estudios para la armonización del sistema de revisión técnica vehicular obligatoria del Distrito Metropoliano de Quito a la nueva Legislación Nacional y a los requerimientos técnicos actuales,» Agencia Metropolitana de Control de Transporte Terrestre, Tránsito y Seguridad Vial del Municipio del Distrito Metropolitano de Quito, Quito, 2017.

[36] T. Khokhar, «Tres grandes ideas para lograr ciudades y comunidades sostenibles,» 31 Enero 2018. [En línea]. Available: http:// www.bancomundial.org/es/news/immersive-story/2018/01/31/3-big-ideas-toachieve-sustainable-cities-and-communities.

[37] M. Bouskela, M. Casseb, S. Bassi, C. De Luca y M. Facchina, «La ruta hacia las Smart Cities, Migrando de una gestión tradicional a la ciudad inteligente, 2016. [En línea]. Available: https://publications. iadb.org/bitstream/handle/11319/7743/ La-ruta-hacia-las-smart-cities-Migrando-de-una-gestion-tradicional-a-la-ciudad-inteligente.pdf. 
[38] prooV, «Smartphones to Smart Cities: How technology is impacting our lives,» 2017. [En línea]. Available: http://proov. io/2016/08/17/smartphones-to-smartcities-how-technology-is-impacting-ourlives/?preview=true.

[39] S. C. ATLAS, «SMART CITIES ATLAS,» 0205 2016. [En línea]. Available: http://www.smartcitiesatlas.com/transporte-y-movilidad-en-una-ciudad-inteligente/.

[40] M. Vicosa, «El independiente,» 29042017. [En línea]. Available: https://www.elindependiente.com/economia/2017/04/29/elreto-del-siglo-xxi-es-mejorar-la-calidadde-vida-en-las-ciudades/.

[41] Metro Ecuador, «metroecuador.com,» 0306 2017. [En línea]. Available: https://www.metroecuador.com.ec/ec/ noticias/2017/07/03/municipio-quito-recogera-datos-planificar-movilidad-smart-city.html.

[42] Alcalde Metropolitano, «Quito será la primera Smart City de Latinoamérica,» 30 Junio 2017. [En línea]. Available: http://prensa.quito.gob.ec/index.php?module $=$ Noticias $\&$ func $=$ news_user view \&i d $=26710 \&$ um $\mathrm{t}=\mathrm{Q}$ uito $\% 20$ ser\%E1\%201a\%20primera\%20Smart\%20 City\%20de\%20Latinoam\%E9rica.
[43] EL Comercio, «Movilidad sostenible para una ciudad moderna,» Tendencias, p. 2, 10 Diciembre 2017.

[44] A. Metropolitana, «quito.gob.ec,» 30 06 2017. [En línea]. Available: http:// prensa.quito.gob.ec/index.php?module $=$ Not i ci as \&f unc $=$ n ews u ser view $\&$ i $d=26710 \& u m t=Q$ uito $\% 20$ ser\%E1\%201a\%20primera\%20Smart\%20 City\%20de\%20Latinoam\%E9rica.

[45] M. A. González, «Criterios de diseño industrial para una propuesta conceptual en el desarrollo de vehículos de los SITP de,» Universidad Nacional de Colombia, Bogotá, 2015.

[46] National Geographic, «Medio Ambiente,» 8 Noviembre 2017. [En línea]. Available: https://www.nationalgeographic.es/medio-ambiente/2017/07/grandes-proyectos-de-transporte-sostenible-para-lucharcontra-los-atascos-en. 